INSTITUT NATIONAL DE RECHERCHE EN INFORMATIQUE ET EN AUTOMATIQUE

\title{
Broadcasting in the Arrowhead Torus
}

Dominique Désérable

N 2827

Mars 1996

THÈME 1 



\title{
RINRIA
}

\section{Broadcasting in the Arrowhead Torus}

\author{
Dominique Désérable* \\ Thème 1 - Réseaux et systèmes \\ Projet API \\ Rapport de recherche $\mathrm{n}^{\circ} 2827$ - Mars 1996 - 15 pages
}

\begin{abstract}
The "arrowhead torus" is a broadcast graph that we define on the 6-valent grid as a Cayley graph. A 3-port wormhole broadcasting protocol is derived first from construction, then improved by using edge-disjoint forests. A store-and-forward broadcasting protocol is derived afterwards, then improved by mixing pipelining and arc-disjoint spanning trees. Costs are given in constant and linear time and compared with lower bounds.
\end{abstract}

Key-words: interconnection networks, Cayley graphs, hexavalent grid, global communications, broadcasting.

also in: CONPAR' 94 - VAPP VI - Lecture Notes in Computer Science 854 - Springer-Verlag

*Institut National des Sciences Appliquées

Campus Universitaire de Beaulieu - 35043 Rennes cedex - France

deserable@irisa.fr 


\section{Diffusion sur le tore en "pointe-de-flèche"}

Résumé : Le tore dit en "sagette" (ou encore "en pointe-de-flèche") est un graphe de diffusion que l'on définit sur la grille 6-valente comme graphe de Cayley. Un protocole 3port de diffusion wormhole est dérivé d'abord par construction, puis amélioré au moyen de forêts arête-disjointes. Un protocole de diffusion store-and-forward est dérivé après-coup, puis amélioré par l'usage conjoint d'un routage pipeline et d'arbres disjoints. Les coûts sont donnés en temps constant et en temps linéaire et comparés aux bornes inférieures.

Mots-clé : réseaux d'interconnexion, graphes de Cayley, grille hexavalente, communications globales, diffusion. 


\section{Introduction}

The choice of interconnection network topologies is a critical subject in the design of efficient distributed memory parallel computers. Most of the performance limitations are due to the performance of the communication system. Between extreme cases such as weakly connected circular rings or strongly connected complete networks, a solution will result from a compromise in order to satisfy, as it was mentioned by Hillis [13], a set of sometimes incompatible requirements : small degree and small diameter, bounded degree and expandability, fault tolerant connectivity and efficient layout, and so forth. Moreover it should be of obvious interest for the routing system that the topology may provide symmetrical schemes for global communications. A symmetrical scheme means that all nodes will behave in a similar way and from this fact will arise a maximum simplicity in the design and processing of the system communication kernel. More precisely, symmetry in the topology means that the representative graph is provided with an algebraic group structure as it is the case for the hypercube and some other families of Cayley graphs [1].

The "arrowhead torus" is a broadcast graph that we define on the 6-valent grid as a Cayley graph. Compared with other more sophisticated interconnection topologies, the grid - and its toroidal extension - has several important advantages such as its simplicity of design and its bounded number of communication links for each processing node. That feature gives a renewal of interest to this ancient topology which had been the first one to be proposed for parallel computers and, in spite of a rather large diameter, returns up-todate $[19,16]$. But the usual "orthogonal" grid in which a vertex has four neighbours (i.e. north-south-east-west) is not unique. It can be shown that there exist exactly three types of regular 2D grids, characterized by their valence (a crystallographic term also used for regular graphs) : thus a regular grid can be either 3 -valent (like in a beehive), 4 -valent (the usual grid) or 6 -valent (for our concern). Note that for a $p$-valent grid, the highest symmetry is obtained when $p=6$; recall that symmetry is an important requirement for interconnection networks, as mentioned above. The reader is referred to [9] for a 2D grid terminology.

Our proposal deals with a recursively scalable torus provided with a self-similar feature: that means that broadcast behaves just like a fractal diffusion process. For a clear distinction from other hexavalent topologies [7, 4], an accurate appellation is needed : we choose in the sequel to name this peculiar one a " 6 -valent arrowhead torus", or arrowhead for short. The reason is highlighted in Fig. 2b. We borrow the term from Mandelbrot who describes one of the Sierpinski famous fractal constructions [15]. As a Cayley graph, the arrowhead torus is generated from an abstract definition (or "presentation") of a discrete group [10]. Given a 3 -fold set of generators corresponding with the three directions of lines in the infinite 6-valent grid, the torus is obtained by superimposing a cyclic relation in the presentation.

In order to avoid to throw the reader into confusion, it should be pointed out that the arrowhead results from an isotropic configuration of the set of generators. As a matter of fact, reversing any generator would yield a non-isotropic, diamond-shaped version of 6 -valent torus. The diamond is closely related to a more usual interconnection topology, the $k$-ary 2 -cube [5]. Indeed the diamond seems no more than a skewed $k$-ary 2-cube with a peculiar value of $k$ and on which a diagonal direction of links would have been added. Nevertheless we 
claim that, by construction, arrowhead and diamond belong to a same family and, moreover, the fact that they are isomorphic is conjectured. The diamond will be presented elsewhere, in a companion paper of reference [10].

We focus presently on the broadcast problem in the arrowhead. Broadcasting (also referred as One-To-All) is the most usual type of global communication encountered in interconnection networks. A message must be sent from one node, called the initiator, to all other nodes in the network. Typically, there exist two species of routing modes : a storeand-forward or packet-switched mode when the message must be stored by intermediate nodes before being forwarded and a wormhole or circuit-switched mode when the source must open a path until destination before sending the message entirely. There are minor differences between wormhole and circuit-switched modes, so our hypotheses will not make distinction between them. For elements of terminology about routing modes the reader is referred to $[3,6,14,21]$. We adopt the conventional linear time statements defined in the literature for a routage from a source to a destination at distance $d: T=d(\beta+L \tau)$ for a store-and-forward routing and $T=\alpha+d \delta+L \tau$ for a wormhole (or wormhole-like) routing, where $\beta$ and $\alpha$ stand for the respective start-up times, $\delta$ stands for the circuit-switching time, and $1 / \tau$ for the bandwidth of the communication link : $\tau$ is thereby the time needed to route a message of unit length, and $L$ will be the length of the message $m$ considered in the sequel. We shall finally assume either a half or full-duplex link capability and a general $k$-port model ( $1 \leq k \leq \Delta$, with $\Delta=6$ for the arrowhead) according to the number of ports simultaneously available. For a survey on modes and models of communication in networks, the reader is referred to [11] and the references therein.

Section 2 gives an elementary description of the arrowhead. In Sect. 3, a wormhole broadcasting protocol is derived first from the algebraic structure of the arrowhead, followed by an improved scheme using edge-disjoint forests. In Sect. 4, a store-and-forward broadcasting protocol is derived afterwards from the wormhole protocol, then improved by mixing pipelining and arc-disjoint spanning trees. Our results may be compared with recent work on the usual [17] (or unusual [18]) - 4-valent - torus. A comparison with existing results for other 6 -valent topologies falls beyond the scope of the present paper and should be examined elsewhere.

\section{The arrowhead 6-valent torus}

Since we focus on the broadcast problem we restrict ourselves to an informal description. We first describe briefly how the arrowhead is generated and display another hexagonal-shaped representation. Then we give a recursive definition of the arrowhead and close the section by setting the basic parameters required for the broadcast problem. The whole is stated without proof, the reader is referred to [10] for detail. 


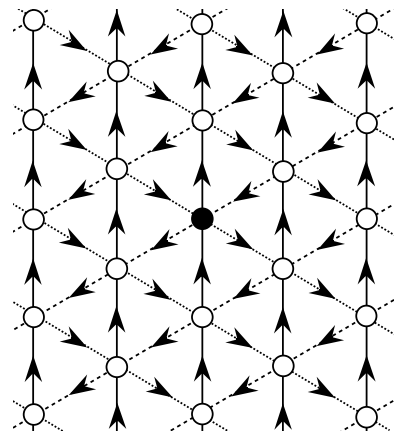

$-a-$

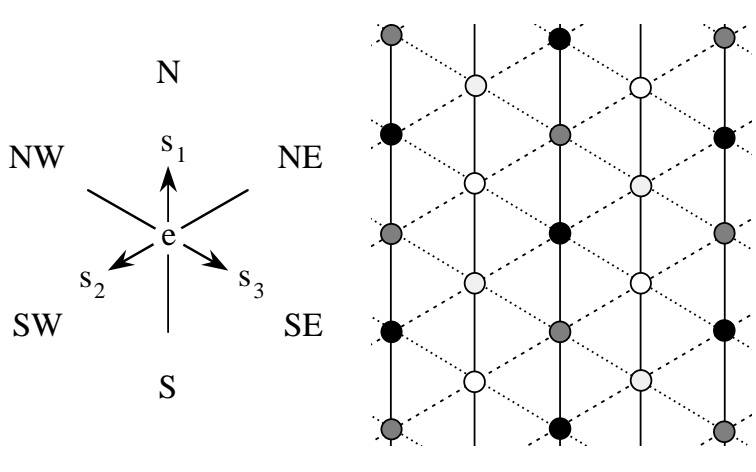

$-b-$

Figure 1: a/ Cayley graph of the group G acting on the vertices of $H$

b/ The cosets of the factor group $G / G_{1}$

\subsection{Generating the arrowhead}

\subsubsection{General conventions}

Although the construction has a combinatorial nature, the arrowhead arises from a metrical definition of the (6-valent) lattice. For convenience, we adopt the orientation $N-N W$-SW-S$S E-N E$ as shown in Fig. 1. The lattice $H=(V, E)$, composed of a set $V$ of vertices and a set $E$ of edges connecting pairs of vertices, is generated by three families of straight lines with respective directions $N-S, S W-N E$ and $S E-N W$. In addition we adopt an arbitrary isotropic orientation for the lines, say $S \rightarrow N, N E \rightarrow S W$ and $N W \rightarrow S E$, or $N-S W-$ $S E$ for short. Note that there exist altogether eight possible orientations, split up into both types, say "isotropic" (such as $N-S W-S E$ and $S-N E-N W$ by a rotation of $\pi$ ) and "anisotropic" (such as $N-N E-N W$ plus five equivalent configurations by $j \pi / 3(j=1, \ldots, 5)$ rotational symmetries). The above metric conventions lead to a Cayley representation [12] for the lattice $H$ in Fig. 1.a with the set of generators $\mathrm{S}=\left\{s_{1}, s_{2}, s_{3}\right\}$. The multiplication on $S$ defines a group $G$ acting on the vertices of $H$, with the set of relations

$$
s_{1} s_{2} s_{3}=e ; \quad s_{1} s_{2} s_{1}^{-1} s_{2}^{-1}=e
$$

where $e$ is the identity. Note that the second relation holds with any combination of elements of $S$ because $G$ is abelian. 


\subsubsection{Generating $\mathcal{A}_{n}$}

We impose now the cyclic relation $R_{n}$ defined by

$$
s_{1}^{2^{n}}=s_{2}^{2^{n}}=s_{3}^{2^{n}}=e
$$

Consider the case $n=1$ and let $G_{1}$ be the set of elements of $G$ that now equal $e$ as a direct consequence. It can be shown that $G_{1}$ is a normal subgroup of $G$ of index 4 and that the cosets, coloured in Fig. 1b, define the factor group

$$
G / G_{1}=\left\{G_{1}, s_{1} G_{1}, s_{2} G_{1}, s_{3} G_{1}\right\} .
$$

The graph of $G / G_{1}$ yields the morphology of the torus $\mathcal{A}_{1}$. In the transformation, one arc in the (oriented) Cayley graph is replaced by one edge. The torus $\mathcal{A}_{1}=\left(V_{1}, E_{1}\right)$ is thus a 2-graph with four vertices, relabelled in the set $I=(0,1,2,3)$ which denotes the respective cosets of the factor group, and is readily 6 -valent.

Roughly speaking there are three ways of generating the arrowhead $\mathcal{A}_{n}$ for any $n$ :

1. Either directly by enumerating the cosets of the factor group induced by the relation $R_{n}$.

2. Recursively from a compound of $\mathcal{A}_{1}$ by $\mathcal{A}_{n-1}$.

3. Or recursively from a compound of $\mathcal{A}_{n-1}$ by $\mathcal{A}_{1}$.

All lead to the normal subgroup $G_{n}$ of index $\left[\mathrm{G}: G_{n}\right]=4^{n}$ and define the torus $\mathcal{A}_{n}=\left(V_{n}, E_{n}\right)$ by turning one arc in the Cayley graph into one edge in the torus as above. For $n>1, \mathcal{A}_{n}$ is a regular 1-graph. The tori are displayed in Fig.2.a for $n=0,1$, 2. Figure 2.b shows the Sierpinski-like arrowhead $\mathcal{A}_{n}$ with $n=7$ arranged from four $\mathcal{A}_{n-1}$ components according to the second method. For clarity, only the metric distribution of the vertex set is drawn.

\subsubsection{Hexagonal representation of the arrowhead}

A holeless, hexagonal arrowhead is depicted in Fig. 2c. It results from an adequate folding of the north, southwest, southeast holey "heads" of the original $\mathcal{A}_{n}$. Schematically, the "area" of $\mathcal{A}_{n}$ is decomposable into nine equilateral triangles as depicted in the pictogram. For simplicity, we call, $T_{1}$ say, such a "head". This northern head is folded once, then translated through the vector $-2^{n} \cdot u_{1}\left(u_{1}\right.$ stands here for the unit vector in the northern direction, associated with the generator $s_{1}$ ), then folded once again to finally fit the $U_{1}$ area. Note that edges are not broken in the transformation. Metrically, the transformation involves a reduction of the average length of "wrapped" edges. Finally, since both graphs are equivalent, we call the transformed one the hexagonal or folded arrowhead $\mathcal{A}_{n}$, or still the arrowhead for short, if it is clear from context. 

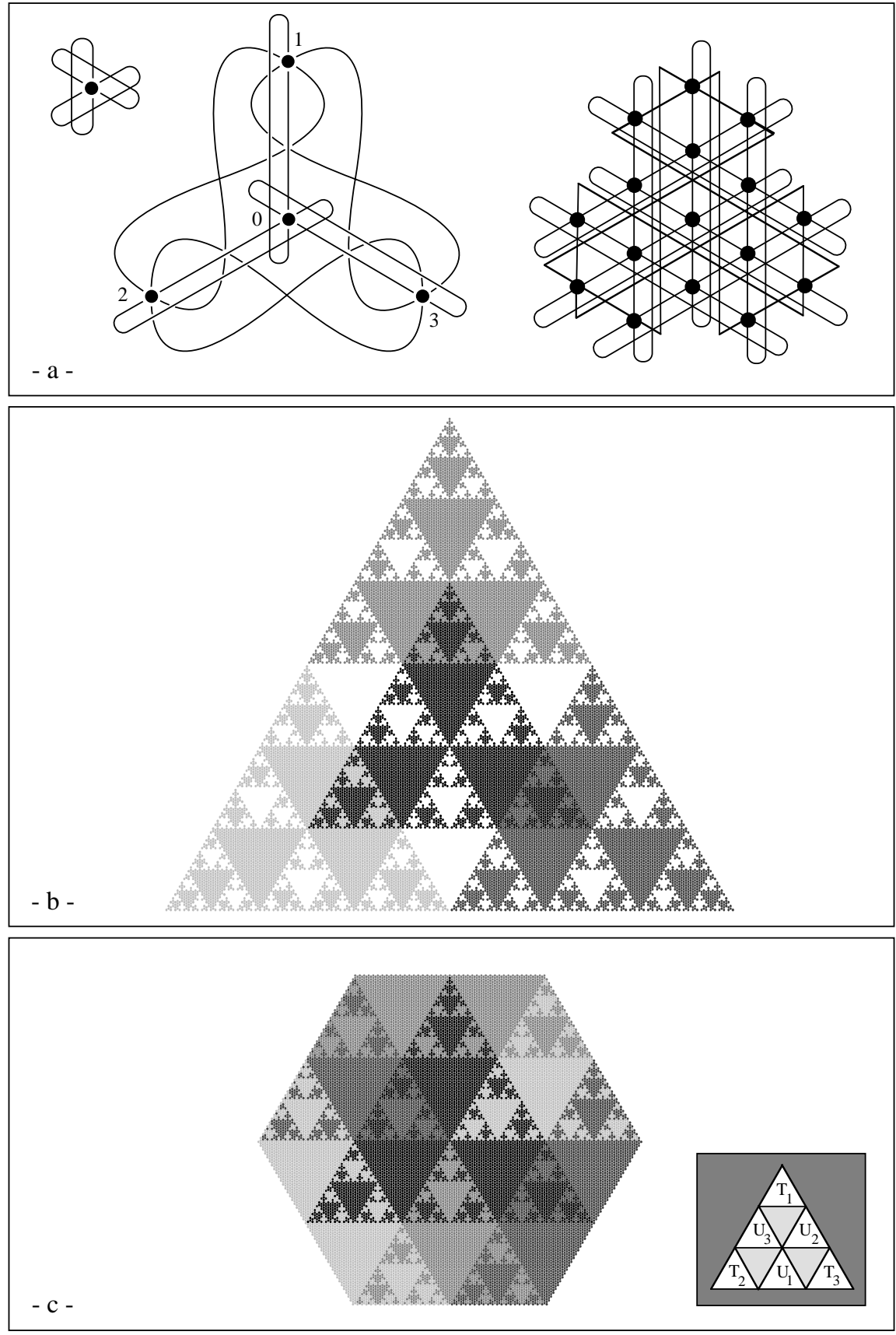

Figure 2: a/ Detail of $\mathcal{A}_{0}, \mathcal{A}_{1}, \mathcal{A}_{2}$

b/ A view of $\mathcal{A}_{7}$ as a compound of $\mathcal{A}_{1}$ by $\mathcal{A}_{6}$

c/ Hexagonal representation of $\mathcal{A}_{7}$

$\operatorname{RR} \mathbf{n}^{\circ} 2827$ 


\subsection{Definition and basic properties}

\subsubsection{Dimensions}

The arrowhead $\mathcal{A}_{n}$ has $N=4^{n}$ vertices and $3 \cdot 4^{n}$ edges.

\subsubsection{Vertices}

Let $I=(0,1,2,3)$ be the ordered set of indices of $V_{1}$ in Fig. 2a. The labelling scheme of $V_{1}$ joined with one or the other recursive compound scheme provides a straightforward quaternary notation with words of $n$ digits $Q_{n}=q_{n-1} q_{n-2} \ldots q_{1} q_{0}$ for numbering the elements of $V_{n}$.

1. Assume first we compose $\mathcal{A}_{1}$ by $\mathcal{A}_{n-1}$. Let ${ }^{i} \mathcal{A}_{n-1}$ be the root, north, southwest or southeast copy of $\mathcal{A}_{n-1}$ respectively for $i=0,1,2$ or 3 . To any vertex labelled $Q_{n-1}=q_{n-2} \ldots q_{1} q_{0}$ in ${ }^{i} \mathcal{A}_{n-1}$ we assign the new word $Q_{n}=i Q_{n-1}$ in $\mathcal{A}_{n}$.

2. Assume now we compose $\mathcal{A}_{n-1}$ by $\mathcal{A}_{1}$. A vertex labelled $Q_{n-1}$ in $\mathcal{A}_{n-1}$ is replaced by the tetrad $\left\{Q_{n}=Q_{n-1} i ; i \in I\right\}$ in $\mathcal{A}_{n}$.

Both schemes are consistent in the sense that the notation will be the same whatever compound alternative we choose. This combinatorial notation allows to bypass any metric notation that would have been defined on the infinite grid. An illustration of the labelling scheme is given in Fig. 3 for $\mathcal{A}_{2}$ and $\mathcal{A}_{3}$. Let us now define in $V_{n}$ the subset :

$$
4^{k} V_{n}=\left\{x \in V_{n}: x \equiv 0\left(\bmod 4^{k}\right)\right\}
$$

for any $k(0 \leq k \leq n)$. Clearly we have : $\left|\mathbf{4}^{k} V_{n}\right|=4^{n-k}$. For the sequel it will be convenient to partition $V_{n}$ into :

$$
V_{n}=\{0\} \cup \bigcup_{p=1}^{n}\left(\mathbf{4}^{n-p} V_{n}-\mathbf{4}^{n-p+1} V_{n}\right)
$$

each of the $n+1$ members being closely related to a normal subgroup of $G$ as defined in Sect. 2.1.

\subsubsection{Edges}

A recursive connecting scheme follows from the relations :

$$
\nu_{0}(0)=0 ; \quad \nu_{n}(4 x)=\nu_{n}^{-1}\left(4 \nu_{n-1}(x)\right)
$$

where $\nu_{k}: V_{k} \rightarrow V_{k}$ defines the neighbour, in a given direction, of any $u$ of $V_{k}$ (clearly $\left.\nu_{k} \cdot \nu_{k}^{-1}(u)=u\right)$. Thus we organize the connection from any element, $x$ say, of $\mathbf{4} V_{n}$ by splitting $E_{n}$ into three parts :

1. a $N-S W-S E$-fold connection : $x$ is connected to the neighbours $x+1, x+2, x+3$. 


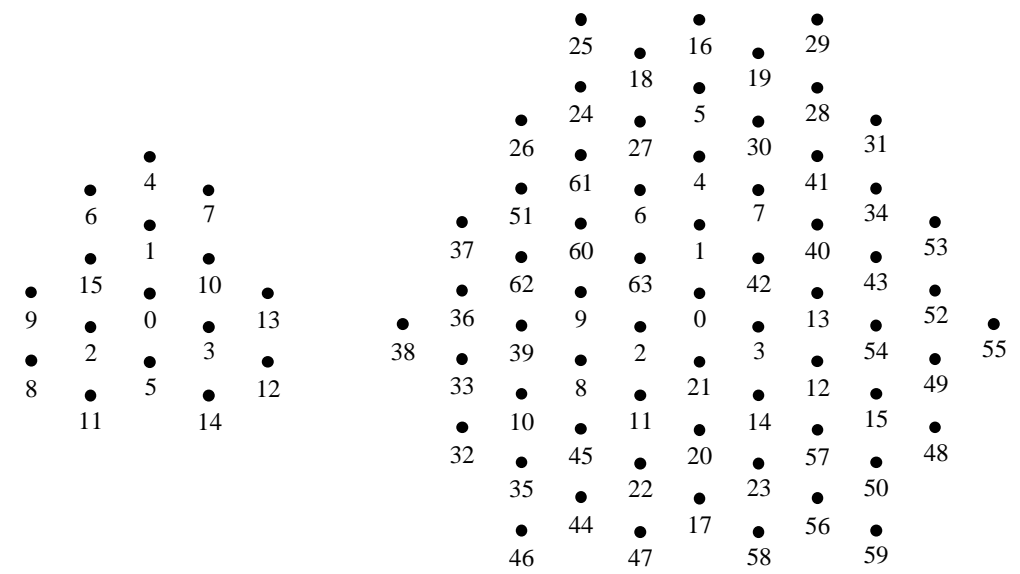

Figure 3: Labelling $\mathcal{A}_{2}$ and $\mathcal{A}_{3}$

2. a $S-N E-N W 3$-fold reversed connection : $x$ is therefore connected to the neighbours one can yield from the above recurrence.

3. a 6 -fold ring surrounding $x$ : for example the $S W$-neighbour of the $N$-neighbour of $x$ is the $N W$-neighbour of $x$ and so forth.

Hence $E_{n}$ is wholly defined since $4^{n-1} 12$-fold disjoint connections are thus achieved.

\subsubsection{Edge-connectivity and diameter}

The arrowhead $\mathcal{A}_{n}$ has an edge-connectivity $\lambda\left(\mathcal{A}_{n}\right)=6$ and a diameter

$$
D_{n}=\frac{2}{3}\left[2^{n}-1\right] \text { or } D_{n}=\frac{2}{3}\left[2^{n}+1\right]-1
$$

depending on whether $n$ is even or odd.

Those definitions will close our informal presentation of the arrowhead torus.

\section{Wormhole Broadcasting}

Since $\mathcal{A}_{n}$ is a Cayley graph, it is vertex-transitive, then without loss of generality we choose the node 0 as initiator in the following. We first present a generic protocol which activates a forest at each timestep, then we give an improved protocol by using two edge-disjoint forests.

\subsection{Generic Protocol}

The wormhole protocol is quite simple. Recall that a way to build $\mathcal{A}_{n}$ is to compose $\mathcal{A}_{1}$ by $\mathcal{A}_{n-1}$. A generic broadcasting scheme, issued from the generation of $\mathcal{A}_{n}$, will follow the 


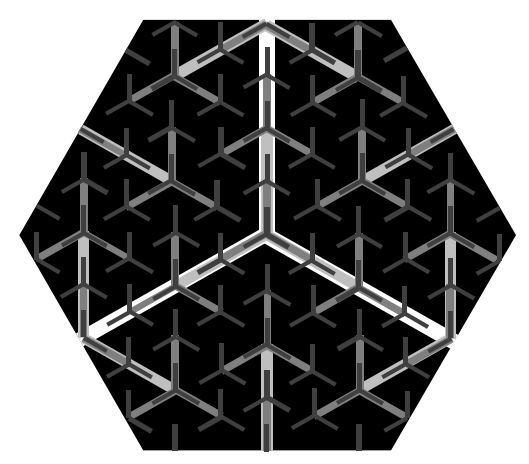

Figure 4: Wormhole "diffusion" : a snapshot

same recursive process. So, broadcasting in $\mathcal{A}_{n}$ must consist in : (i) routing the message from the initiator to the centroid (say, a "descendant") of any $i A_{n-1}\left(i \in I^{\star} ; I^{\star}=I-\{0\}\right)$ if any : shortly we will sometimes say that the initiator "diffuses" (see the snapshot in Fig. 4) ; (ii) broadcasting in $\mathcal{A}_{n-1}$. The process readily leads to a wormhole protocol, expressed according to the 4-ary notation of $V_{n}$ as follows.

Lemma 1 At any timestep $p(1 \leq p \leq n)$ there exists one forest, whose trees are rooted in $W_{n, p}=\mathbf{4}^{n-p+1} V_{n}$, informing the set $\overline{\mathbf{4}}^{n-p} V_{n}-W_{n, p}$ of the message.

Proof Let $\varepsilon_{n, k}$ be a nul word of $n-k$ digits. The proof is immediate, since with the above scheme :

1. At step 1, the initiator $\varepsilon_{n, 0}$ informs its triad : $\left(q_{n-1} \varepsilon_{n, 1}\right) \quad \forall q_{n-1} \in I^{\star}$.

2. At step $(p>1)$, any informed node $\left(q_{n-1} \ldots q_{n-p+1} \varepsilon_{n, p-1}\right) \quad\left(\forall k: q_{k} \in I\right)$ will inform its triad : $\left(q_{n-1} \ldots q_{n-p} \varepsilon_{n, p}\right) \quad \forall q_{n-p} \in I^{\star}$.

In other words the broadcast graph at step $p$ is a forest composed of $4^{p-1}$ balanced trees of depth $2^{n-p}$.

Proposition 2 There exists a 3-port half-duplex wormhole protocol to broadcast in $\frac{1}{2} \log _{2} N$ steps and whose time is

$$
{ }^{w} b_{H_{3}}\left(\mathcal{A}_{n}\right)=\frac{1}{2}(\alpha+L \tau) \log _{2} N+(\sqrt{N}-1) \delta .
$$

Proof First, the number of steps gives the cost in the constant time model. It is clear that the whole broadcast is completed in $n=\log _{4} N$ steps and uses $n$ time-disjoint forests : let us observe, in particular, that the initiator diffuses $n$ times, by successive waves of decreasing 
amplitude. In linear time now, let $T_{k}$ be the time required to broadcast in any $\mathcal{A}_{k}$. Clearly:

$T_{1}=\alpha+\delta+L \tau$ then assume by induction that :

$T_{n-1}=(n-1)(\alpha+L \tau)+\left(2^{n-1}-1\right) \delta$

Remains to examine the first step, i.e., the initiator of $\mathcal{A}_{n}$ must diffuse towards its descendants. Observe that they are at distance $2^{n-1}$. Hence sequentially we have: a start-up, $2^{n-1}$ switches to cut through, then the message to send. Therefore :

$$
T_{n}=\left(\alpha+2^{n-1} \delta+L \tau\right)+T_{n-1}=n(\alpha+L \tau)+\left(2^{n}-1\right) \delta .
$$

\subsection{Improved Wormhole Broadcasting with Edge-disjoint Forests}

Because the previous protocol broadcasts through no more than three ports, it can be easily improved by using a sequence of edge-disjoint forests in opposite directions. Due to the symmetry of the above broadcast pattern, it can be rotated through $\pi$ : one forest broadcasts along $N-S W-S E$ directions as above, while the other will broadcast along $S-N E$ $N W$ directions simultaneously. We split the message $m$ into two half submessages $m_{1}$ and $m_{2}$.

Lemma 3 At any timestep $p(1 \leq p \leq n)$ there exist two edge-disjoint forests, whose respective trees are rooted in $W_{n, p}$, informing the set $\mathbf{4}^{n-p} V_{n}-W_{n, p}$ of both $m_{1}$ and $m_{2}$ submessages.

Proof If $W_{n, p}^{\prime}$ and $W_{n, p}^{\prime \prime}$ are the respective sources of both symmetric patterns, since $W_{n, 0}^{\prime}=W_{n, 0}^{\prime \prime}=\{0\}$ an inductive proof can readily show that the informed sets at step $p$ still coincide. Moreover, the shortest distance between two sources is $2^{n-p+1}$, that is twice the depth of trees, consequently paths are necessarily edge-disjoint.

Corollary 4 There exists a 6-port half-duplex wormhole protocol to broadcast in $\frac{1}{2} \log _{2} N$ steps and whose time is

$$
{ }^{w} b_{H_{*}}\left(\mathcal{A}_{n}\right)=\frac{1}{2}\left(\alpha+\frac{L \tau}{2}\right) \log _{2} N+(\sqrt{N}-1) \delta .
$$

Note that if in real cases $\delta \ll \alpha$ is a realistic assumption, then the time needed to broadcast long messages grows with $\log _{2} N$. The above result should be compared with the lower bound given in [18] for a vertex-transitive graph $G$ with degree $\Delta$ and diameter $D$ :

$$
{ }^{w} b_{F_{*}}(G) \geq \max \left(\alpha\left\lceil\log _{\Delta+1} N\right\rceil, \alpha+D \delta+\frac{L \tau}{\Delta}\right)
$$

giving for the arrowhead :

$$
{ }^{w} b_{F_{*}}\left(\mathcal{A}_{n}\right) \geq \max \left(\mu \cdot \alpha \log _{2} N, \alpha+\delta \cdot \frac{2}{3} \sqrt{N}+\frac{L \tau}{6}\right)
$$

with the constant factor $\mu \sim 0.36$; (we estimate besides that the diameter is close to $\frac{2}{3} \cdot \sqrt{N}$, whether $n$ is even or odd, according to (7)). 


\section{Store-and-Forward Broadcasting}

The previous wormhole scheme is derived directly from the recursive structure of the arrowhead, but it neither takes advantage of the fact that each node must receive a copy of the broadcast message, nor allows pipelining. In the following, store-and-forward protocols are derived from the generic wormhole scheme : the first one by a direct transformation of the generic wormhole protocol, the second one by using two edge-disjoint spanning trees and the third one by mixing pipelining.

\subsection{Derivation of the Generic Wormhole Protocol}

Proposition 5 There exists a 3-port half-duplex store-and-forward protocol to broadcast in $(\sqrt{N}-1)$ steps and whose time is :

$$
{ }^{s f} b_{H_{3}}\left(\mathcal{A}_{n}\right)=(\sqrt{N}-1)(\beta+L \tau) .
$$

Proof Let $T_{k}$ be the constant time required to broadcast in any $\mathcal{A}_{k}$. Clearly $T_{1}=1$. Assume we compose $\mathcal{A}_{1}$ by $\mathcal{A}_{n-1}$ and, by induction, that a spanning tree broadcasts in $\mathcal{A}_{n}-1$ in time : $T_{n-1}=2^{n-1}-1$. By symmetry, we can restrict ourselves to the northern branch of $\mathcal{A}_{n}$. To achieve the complete broadcast, first we broadcast in ${ }^{0} \mathcal{A}_{n-1}$, then we route from the northern leaf of ${ }^{0} \mathcal{A}_{n-1}$ to the root of ${ }^{1} \mathcal{A}_{n-1}$, finally we terminate the broadcast in ${ }^{1} \mathcal{A}_{n-1}$. Therefore, a spanning tree broadcasts in $\mathcal{A}_{n}$ in the constant time : $T_{n}=2 T_{n-1}+1=2^{n}-1$. In linear time we have just to observe that the cost of one step is $(\beta+L \tau)$.

\subsection{Improved Store-and-Forward Broadcasting with Edge-disjoint Spanning Trees}

Lemma 6 There exist two edge-time disjoint spanning trees whose depth is $(\sqrt{N}-1)$.

Proof Clearly we have two arc-disjoint spanning trees, since both opposite oriented patterns $N-S W-S E$ and $S-N E-N W$ cannot overlap, and we can derive a straigthforward 6-port full-duplex protocol from Proposition 5. More thoroughly, we can prove that a half-duplex protocol remains valid with the same cost provided that the whole process is synchronized. We decompose $V_{n}$ into the subsets $\mathbf{4} V_{n}$ and $V_{n}-\mathbf{4} V_{n}$ and label the arcs of both spanning trees in constant time. The root "shouts" the message $m$ at step 1 by diffusing submessages $m_{1}$ and $m_{2}$ through $N-S W-S E$ and $S-N E-N W$ respectively. Observe that a vertex of $\mathbf{4} V_{n}$ receives at even steps then diffuses at odd steps whereas a vertex of $V_{n}-\boldsymbol{4} V_{n}$ receives at odd steps then forwards -if needed- at even steps. Consequently, any edge carries at most two distinct timestep numbers upon completion. 
Corollary 7 There exists a 6-port half-duplex store-and-forward protocol to broadcast in $(\sqrt{N}-1)$ steps and whose time is :

$$
{ }^{s f} b_{H_{*}}\left(\mathcal{A}_{n}\right)=(\sqrt{N}-1)\left(\beta+\frac{L \tau}{2}\right) .
$$

\subsection{Mixing Pipelining and Edge-disjoint Spanning Trees}

The above result can still be improved for long messages by using the technique of pipelining introduced by Saad and Schultz in [20]. The message is split into packets of equal size and a critical size is computed in order to achieve an optimal cost. In particular, given a path of length $h$ between source and destination, packets are sent and forwarded one by one right away, with a resulting time upon completion of : $(\sqrt{(h-1) \beta}+\sqrt{L \tau})^{2}$. Pipelining has been generalized afterwards under link-bound model in which case $h$ stands for the depth of a spanning tree [2].

Corollary 8 There exists a 6-port full-duplex store-and-forward protocol whose time is:

$$
{ }^{s f} b_{F_{*}}\left(\mathcal{A}_{n}\right)=\left(\sqrt{(\sqrt{N}-2) \beta}+\sqrt{\frac{L \tau}{2}}\right)^{2}
$$

Proof If $h(p)$ is the maximum depth of $p$ arc-disjoint spanning trees there exists a protocol for a link-bound broadcasting with time: $\left(\sqrt{(h(p)-1) \beta}+\sqrt{\frac{L \tau}{p}}\right)^{2}$. With pipelining we should release the half-duplex capability of Corollary 7 because the spanning trees remain arc-disjoint but cannot be edge-time disjoint any more. Setting $p=2$ and $h(p)=\sqrt{N}-1$ in the above formula gives the result.

The result of Corollary 8 should be compared with the lower bound given in [11] for a regular graph $G$ :

$$
{ }^{s f} b_{F_{*}}(G) \geq D(\beta+\tau)+(L-\Delta) \frac{\tau}{\Delta}
$$

giving for the arrowhead :

$$
{ }^{s f} b_{F_{*}}\left(\mathcal{A}_{n}\right) \geq \frac{2}{3} \sqrt{N}(\beta+\tau)+(L-6) \frac{\tau}{6}
$$

with the same minor approximation for the diameter as in the wormhole case.

\section{Conclusion}

This paper strengthens a first presentation of the "arrowhead torus", a new interconnection topology generated on the hexavalent grid [10]. Generic schemes for broadcasting have 
shown to be simple and efficient at once, running through a circuit-switched mode or a packet-switching mode as well. From the organization of the paper, it must be clear that the good behaviour the arrowhead has towards the broadcast problem results directly from the symmetry of the algebraic structure whose it is provided as a Cayley graph. The question of possible improvements of those algorithms is settled now as an open problem while relevant schemes with regard to other types of global communications are investigated in a more general framework.

Acknowledgments A flashback to a prior statement of the problem [8] reveals that the RUMEUR Summer School' 92 held in Cargese (Corse) was undoubtedly fruitful to the author.

\section{References}

[1] S.B. Akers, B. Krishnamurthy : A group-theoretic model for symmetric interconnection networks. IEEE Trans. Comp. 38 (4) (1989) 555-66

[2] J.C. Bermond, P. Fraignaud : Communication in interconnection networks. Proc. Combinatorial Optimization in Science and Technology' 91 (1991)

[3] J.C. Bermond, M. Syska : Routage "wormhole" et canaux virtuels. Rapport de recherche $\mathrm{n}^{\circ}$ 92-07 I3S Nice (1992)

[4] M.S. Chen, K.G. Shin, D.D. Kandlur : Addressing, routing, and broadcasting in hexagonal mesh multiprocessors. IEEE Trans. Comp. 39 (1) (1990) 10-18

[5] W.J. Dally : Performance analysis of $k$-ary $n$-cube interconnection networks. IEEE Trans. Comp., C-39 (6) (1990) 775-85

[6] W.J. Dally, C.L. Seitz : Deadlock-free message routing in multiprocessor interconnection networks. IEEE Trans. Comp., C-36 (5) (1987) 547-53

[7] A.L. Davis, S.V. Robison : The architecture of the FAIM-1 symbolic multiprocessing system. Proc. 9-th Int. Joint. Conf. on Artificial Intelligence (1985) 32-38

[8] D. Désérable : Diffusion on scalable honeycomb networks. Publication interne ${ }^{\circ} 655$ IRISA Rennes (1992)

[9] D. Désérable : A terminology for 2D grids. Publication interne ${ }^{\circ} 839$ IRISA Rennes (1994)

[10] D. Désérable : The arrowhead torus : a Cayley graph on the 6-valent grid. Publication interne $\mathrm{n}^{\circ} 840$ IRISA Rennes (1994)

[11] P. Fraignaud, E. Lazard : Methods and problems of communication in usual networks. Special Issue of Discrete Applied Math. on Broadcasting (to appear) 
[12] I. Grossman, W. Magnus : Groups and their graphs. New Mathematical Library $n^{\circ} 4$, Random House, New-York (1964)

[13] D. Hillis : The Connection Machine. MIT Press (1985)

[14] P. Kermani, L. Kleinrock : Virtual cut-through : a new computer communication switching technique. Computers Networks 3 (1979) 267-86

[15] B.B. Mandelbrot : The fractal geometry of nature. Freeman and Cie, San Francisco (1982)

[16] D. May : The next generation transputers and beyond. Distributed Memory Computing, Lecture Notes in Computer Science 487, Springer-Verlag (1991) 7-22

[17] P. Michallon, J.C. Bermond, D. Trystram : Broadcasting on wraparound meshes with parallel monodirectional links. Parallel Computing 18 (1992) 639-48

[18] J.G. Peters, M. Syska : Circuit-switched broadcasting in torus networks. CMPT TR n 93-04, Simon Fraser Univ., Burnaby, Canada (1993)

[19] J. Rattner : The new age of supercomputing. Distributed Memory Computing, Lecture Notes in Computer Science 487 Springer-Verlag (1991) 1-6

[20] Y. Saad, M.H. Schultz : Data communication in parallel architectures. Parallel Computing 11 (2) (1989) 131-50

[21] S.R. Seidel : Circuit-switched vs. store-and-forward solutions to symmetric communication problems. Proc. 4-th Conf. on Hypercube Concurrent Computers and Applications (1989) 253-55 


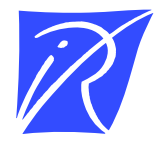

Unité de recherche INRIA Lorraine, Technopôle de Nancy-Brabois, Campus scientifique,

615 rue du Jardin Botanique, BP 101, 54600 VILLERS LÈS NANCY

Unité de recherche INRIA Rennes, Irisa, Campus universitaire de Beaulieu, 35042 RENNES Cedex

Unité de recherche INRIA Rhône-Alpes, 46 avenue Félix Viallet, 38031 GRENOBLE Cedex 1

Unité de recherche INRIA Rocquencourt, Domaine de Voluceau, Rocquencourt, BP 105, 78153 LE CHESNAY Cedex

Unité de recherche INRIA Sophia-Antipolis, 2004 route des Lucioles, BP 93, 06902 SOPHIA-ANTIPOLIS Cedex

Éditeur

INRIA, Domaine de Voluceau, Rocquencourt, BP 105, 78153 LE CHESNAY Cedex (France)

ISSN 0249-6399 